\title{
Completeness of Neighbourhood Logic
}

\author{
Rana Barua ${ }^{1 \star}$, Suman Roy ${ }^{\star \star \star}$, and Zhou Chaochen ${ }^{3 \star \star \star}$ \\ 1 Stat-Math. unit, I.S.I., 203 B.T. Road, Calcutta 700 035, India. \\ rana@isical.ac.in \\ 2 Computer Science and Automation, I.I.Sc., Bangalore 560 012, India. \\ suman@csa.iisc.ernet.in, corresponding author \\ 3 International Institute for Software Technology, UNU/IIST, P.O. Box 3057, Macau \\ zcc@iist.unu.edu
}

\begin{abstract}
This paper presents a completeness result for a first-order interval temporal logic, called Neighbourhood Logic (NL) which has two neighbourhood modalities. NL can support the specification of liveness and fairness properties of computing systems as well as formalisation of many concepts of real analysis. These two modalities are also adequate in the sense that they can derive other important unary and binary modalities of interval temporal logic. We prove the completeness result for NL by giving a Kripke model semantics and then mapping the Kripke models to the interval models for NL.
\end{abstract}

\section{Introduction}

In many applications, digital systems reacting with environment and events have to produce an output before a certain delay has elapsed. Time requirements - both qualitative as well as quantitative - have to be considered to reason about such systems. Thus, for such purposes one has to consider a real-time logic. Various such logics have been proposed. Some of these formalisms interpret formulas over intervals of time [5]11]16]17!19]; notably among them are Interval Temporal Logic (ITL) [11] and Duration Calculus (DC) [619]. ITL is a firstorder interval modal logic which uses a binary modal operator " $\frown$ " which is interpreted as the operation of "chopping" an interval into two parts. DC is an extension of ITL in the sense that temporal variables are written in the form of the integrals of "states".

Since chop " $\sim$ " is a contracting modality, ITL-based logics can succintly express properties of the real-time systems, such as; "for all time intervals of a given length, $\phi$ must be true", or "if $\phi$ holds for a time interval, then there is a sub-interval where $\psi$ holds". However, these logics cannot express liveness properties, which depend on intervals lying outside the reference interval, like;

* The work was done when the author visited UNU/IIST as a fellow during MayAug.'97

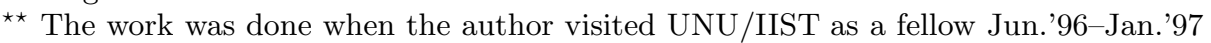

$\star \star \star$ On leave of absence from Software Institute, Chinese Academy of Sciences 
"eventually there is an interval where $\phi$ is true, and " $\phi$ will hold infinitely often in the future".

Another limitation of these logics is that when they are used in the specification of hybrid systems the notions of real analysis such as limit, continuity and differentiability cannot be suitably formalised in them. These notions are neighbourhood properties of a point which cannot be defined in those logics. Although an informal mathematical theory of calculus can be assumed as in extended duration calculus [20, Hybrid Statecharts [10], Hybrid Automata [1] and $\mathrm{TLA}^{+}$[9], a formalization of real analysis may help in developing theorem provers for supporting the design of hybrid systems.

In order to improve the expressiveness of ITL, expanding modalities have been used. Venema [17] gives a complete axiomatization of a propositional calculus with three binary modalities; in addition to chop (designated as C) it has modalities $T$ and $D$, which can represent properties outside the interval. Some of the axioms and rules in it are quite complicated. Other expanding modalities which are unary have been considered in Halpern and Shoham [5]. But many notions of real analysis cannot be formalised without first-order quantifiers.

In [18], Zhou and Hansen proposed a first-order interval logic called Neighbourhood Logic (NL) which has provisions for specifying liveness and fairness properties as well as formalising some notions in real analysis. This logic has two expanding modalities $\diamond_{l}$ and $\diamond_{r}$, called the left and right neighbourhood modality respectively. These modalities refer to some past and future intervals of time respectively with respect to the original interval of time being observed.

Although, it is not very hard to see that the Propositional NL is complete with respect to Kripke models, it seems to be quite inadequate to derive the modalities considered in [517] (but not conversely). Moreover, Propositional NL forms a fragment of the complete logic proposed by Venema in [16]. Nevertheless, the adequacy of the neighbourhood modalities can be established by deriving the other unary and binary modalities of [5] in a first-order logic of the neighbourhood modalities and the interval length (cf.[18]). Thus first-order Neighbourhood Logic seems to have more expressive power than those of 4 4,517] with a minimum number of modalities.

This paper presents the syntax and semantics of first-order Neighbourhood Logic and then establishes a completeness result. First, we establish a completeness theorem for the NL formulas in the Kripke model (or possible world model). Then we map the Kripke model to the interval model and prove the completeness of NL in the interval model. Dutertre 4] has proved a similar completeness result for ITL with chop modality. Both follow the approach suggested by [817].

\section{$2 \quad$ Neighbourhood Logic (NL)}

\subsection{Syntax of NL}

A language $\mathcal{L}$ for NL consists of an infinite collection of global variables, $\mathbf{V} \widehat{=}\{x, y$, $z, \ldots\}$ and also an infinite collection of temporal variables, $\mathbf{T} \widehat{=}\left\{\ell, v_{1}, v_{2}, \ldots\right\}$, 
where $\ell$ is a special symbol which will denote the length of an interval. " $\ell$ " will depict the "natural" properties of length in the axioms of the logic to be introduced later. In addition, the language contains an infinite set of global function symbols $\mathbf{F}$ and global predicate symbols $\mathbf{H}$. These symbols are called global because their meaning will be independent of time. With each of the function and predicate symbols is associated an arity $n \geq 0$. Function symbols of arity 0 will be called constants. Predicate symbols of arity 0 are propositions which include two Boolean symbols true and false. F includes the symbols,+- and $\mathbf{H}$ includes $=, \geq$ etc. There is also an infinite set of temporal propositional letters $\mathbf{P} \widehat{=}\{X, Y, \ldots\}$ which will be interpreted as Boolean-valued functions on intervals. The vocabulary also consists of propositional connectives $\neg$ and $\vee$, the existential quantifier $\exists$ and the left neighbourhood modality $\diamond_{l}$ and the right neighbourhood modality $\diamond_{r}$. The other usual connectives $\wedge, \Rightarrow, \Leftrightarrow$ as well as the universal quantifier $\forall$ are introduced as abbreviations.

The terms denoted as $\theta, \theta_{i}$, are defined by the following abstract syntax:

$\theta::=x|\ell| v \mid f^{n}\left(\theta_{1}, \ldots, \theta_{n}\right) ; \quad x \in \mathbf{V}, \quad v \in \mathbf{T}, \quad f \in \mathbf{F}$.

The formulas, denoted as $\phi, \psi$, are defined by the following abstract syntax:

$\phi::=X\left|G^{n}\left(\theta_{1}, \ldots, \theta_{n}\right)\right| \neg \phi|\phi \vee \psi|(\exists x) \phi\left|\diamond_{l} \phi\right| \diamond_{r} \phi ; \quad x \in \mathbf{V}, \quad X \in$ $\mathbf{P}, \quad G \in \mathbf{H}$.

A term is global or rigid, if it does not contain any temporal variables. A formula is global or rigid, if it does not contain any temporal variables, any temporal propositional letters, or any neighbourhood modalities.

\subsection{Semantics of NL}

We fix our domain to be a non-empty set $\mathbb{D}$ (containing the constant symbol 0 ) which will be the underlying representation of time as well as lengths of intervals. Traditional semantics, however, distinguishes between temporal domain $T$ (which is generally a totally ordered set) and duration domain $\mathbb{D}$ which represents durations of time intervals. The duration domain satisfies certain constraints since their elements are supposed to measure "lengths" of time intervals(cf. [4]). Here, for simplicity, we take the time domain to be the same as the duration domain. As in [4], we want $\mathbb{D}$ to have certain properties which are specified by the following axioms.

D 1 Axioms for =:

The standard axioms for $=$ are assumed ( $c f .[15)$.

D 2 Axiom for + :
1. $x+0=x$.
3. $x+(y+z)=(x+y)+z$.
2. $x+y=y+x$.
4. $(x+y=x+z) \Rightarrow y=z$.

D 3 Axioms for $\geq$ :

1. $0 \geq 0$.

2. $x \geq 0 \wedge y \geq 0 \Rightarrow x+y \geq 0$.

3. $x \geq y \Leftrightarrow \exists z \geq 0 .(x=y+z)$.

where we write $y>x$ if $(y \geq x) \wedge(y \neq x)$.

D 4 Axioms for - :

1. $x-y=z \Leftrightarrow x=y+z$. 
Clearly, $\mathbb{R}$ (the reals), $\mathbb{Q}$ (the rationals) and $\mathbb{Z}$ (the integers) are examples of domains which satisfy the above axioms. From the above axioms it can be shown that $(\mathbb{D},+)$ is a commutative group with 0 as the additive identity and $-y$ as the additive inverse of $y$.

The time domain is $\mathbb{D}$ and the set of all intervals $I I$ is given by:

$$
\text { II } \widehat{=}\{[a, b]: a, b \in \mathbb{D} \text { and }(b \geq a)\},
$$

where the interval $[a, b]$ is defined as

$$
[a, b] \widehat{=}\{x \in \mathbb{D}: b \geq x \geq a\} .
$$

The global variables are assigned meaning through a valuation or value assignment $\quad \nu: \mathbf{V} \rightarrow \mathbb{D}$. Given an interval $[a, b] \in \mathbb{I}$ the meaning of the temporal variables, propositional letters, function and predicate symbols is given by an interpretation function $\mathcal{I}$ such that,

1. $\mathcal{I}(0,[a, b])=0$,

2. $\mathcal{I}(\ell,[a, b])=b-a$,

3. $\mathcal{I}(v,[a, b]) \in \mathbb{D}$; for $v \in \mathbf{T}$,

4. $\mathcal{I}(X,[a, b]) \in\{\mathrm{tt}, \mathrm{ff}\}$ for $X \in \mathbf{P}$,

5. $\mathcal{I}(f,[a, b])=\underline{f}$, for an $n$-ary function $f \in \mathbf{F}$,

where $f: \mathbb{D}^{n} \rightarrow \mathbb{D}$ is any standard interpretation of $f$,

6. $\mathcal{I}(G,[a, b])=\underline{G}$, for an $n$-ary predicate symbol $G \in \mathbf{H}$, where $\underline{G}: \mathbb{D}^{n} \rightarrow\{\mathrm{tt}, \mathrm{ff}\}$ is any standard interpretation of $G$.

Note that " + " and " $-"$ " are interpreted as the associated binary operations on ID.

Given a valuation $\nu$, the terms are interpreted in the usual way by induction on the length of terms [15].

We shall call the pair $\mathcal{M} \widehat{=}\langle\mathbb{D}, \mathcal{I}\rangle$ an interval model. Let $\mathcal{M}, \nu,[a, b] \models A$ denote that the formula $A$ is satisfied in the interval $[a, b]$ (also called the reference interval) with respect to the model $\mathcal{M}$ and valuation $\nu$. Satisfiability can then be defined by induction on the formulas in a standard way [15]8]. We only state the cases for formulas with modalities $\diamond_{l}$ and $\diamond_{r}$.

1. $\mathcal{M}, \nu,[a, b] \models \diamond_{l} A$ iff there exists $c, a \geq c$ such that $\mathcal{M}, \nu,[c, a] \models A$.

2. $\mathcal{M}, \nu,[a, b] \models \diamond_{r} A$ iff there exists $d, d \geq b$ such that $\mathcal{M}, \nu,[b, d] \models A$.

We say that $A$ is valid, written as $\models A$, iff for any model $\mathcal{M}$, any valuation $\nu$ and interval $[a, b], \mathcal{M}, \nu,[a, b] \models A$. Also $A$ is satisfiable iff for some model $\mathcal{M}$, valuation $\nu$, and some interval $[a, b], \mathcal{M}, \nu,[a, b] \models A$.

\section{The Proof System for NL}

In the following set of axioms and rules (as well as elsewhere), $\diamond(\square)$ can be instantiated by either $\diamond_{l}$ or $\diamond_{r}\left(\square_{l}\right.$ or $\square_{r}$ respectively). The following abbreviation will be adopted.

$$
\begin{aligned}
& \bar{\diamond} \hat{=}\left\{\begin{array}{l}
\diamond_{r}, \text { if } \diamond=\diamond_{l} \\
\diamond_{l}, \text { if } \diamond=\diamond_{r}
\end{array}\right. \\
& \square \hat{=} \neg \diamond \neg \\
& \bar{\square} \widehat{=} \neg \diamond \neg \\
& \diamond^{c} \widehat{=} \bar{\diamond}
\end{aligned}
$$




\section{Axioms}

A1 Global formulas are not connected to intervals.

$\diamond A \Rightarrow A$, provided $A$ is a global formula.

A2 Interval length is non-negative.

$\ell \geq 0$

A3 Neighbourhood can be of arbitrary length.

$x \geq 0 \Rightarrow \diamond(\ell=x)$

A4 Neighbourhood modalities can be distributed over disjunction and existential quantifier.

$$
\begin{aligned}
& \diamond(A \vee B) \Rightarrow \diamond A \vee \diamond B \\
& \diamond \exists x . A \quad \Rightarrow \exists x . \diamond A
\end{aligned}
$$

(The second part of $\mathbf{A 4}$ implies that the analogue of Barcan Formula is true.)

A5 A left (right) neighbourhood coincides with any other left (right) neighbourhood provided they have the same length. In other words, neighbourhood is determined by its length.

$\diamond((\ell=x) \wedge A) \Rightarrow \square((\ell=x) \Rightarrow A)$

A6 Left (right) neighbourhoods of an interval always start at the same point.

$\diamond \bar{\diamond} A \Rightarrow \square \bar{\diamond} A$

A7 Left (right) neighbourhood of the ending (beginning) point of an interval is the interval itself, if it has the same length as the interval.

$(\ell=x) \Rightarrow\left(A \Leftrightarrow \diamond^{c}((\ell=x) \wedge A)\right)$

A8 Two consecutive left (right) expansions can be replaced by a single left (right) expansion, if the third expansion has a length of the sum of the first two.

$((x \geq 0) \wedge(y \geq 0)) \Rightarrow(\diamond((\ell=x) \wedge \diamond((\ell=y) \wedge \diamond A)) \Leftrightarrow \diamond((\ell=x+y) \wedge \diamond A))$

Rule schemas

$M$ (Monotonicity) If $\phi \Rightarrow \psi \quad$ then $\diamond \phi \Rightarrow \diamond \psi$.

$\mathrm{N}$ (Necessity) If $\phi \quad$ then $\square \phi$.

MP (Modus Ponens) If $\phi$ and $\phi \Rightarrow \psi$ then $\psi$.

$\mathrm{G}$ (Generalization) If $\phi \quad$ then $(\forall x) \phi$.

The proof system also contains axioms D1-D4 and axioms of propositional logic and first-order predicate logic. They can be taken as any complete system for first-order logic except for some restrictions on the instantiation of quantified formulas. A term $\theta$ is called free for $x$ in $\phi$ if $x$ does not occur freely in $\phi$ within a scope of $\exists y$ or $\forall y$, where $y$ is any variable occurring in $\theta$. We also adopt the following axioms:

$$
\begin{aligned}
& \forall x . \phi(x) \Rightarrow \phi(\theta) \\
& \phi(\theta) \Rightarrow \exists x . \phi(x)
\end{aligned} \quad\left(\begin{array}{l}
\text { if either } \theta \text { is free for } x \text { in } \phi(x) \text { and } \theta \text { is rigid } \\
\text { or } \theta \text { is free for } x \text { in } \phi(x) \text { and } \phi(x) \text { is modality free. }
\end{array}\right)
$$

A proof of an NL formula $A$ is a finite sequence of NL formulas $A_{1}, \ldots, A_{n}$, where $A_{n}$ is $A$, and each $A_{i}$ is either an instance of one of the axiom schemas mentioned 
above or obtained by applying one of the inference rules, also mentioned above, to the previous members of the sequence. We write $\vdash A$ to mean that there exists a proof of $A$ in NL and we say that $A$ is a theorem in NL (or $A$ is provable in NL).

The following is easy to check by induction on the length of proof.

Theorem 1 ((Soundness in NL)). An NL formula which can be proved in the calculus must be valid (in any interval model).

\section{Kripke Completeness}

Kripke Model A Kripke model $\mathcal{K}$ for NL is a quintuple $\left\langle W, R_{l}, R_{r}, \mathbb{D}, \mathcal{I}\right\rangle$, where

- $W$ is a non-empty set of possible worlds,

- $R_{l}$ and $R_{r}$ are binary relations on $W$, called accessibility relations,

- $\mathbb{D}$ is a non-empty set, called the domain,

- $\mathcal{I}$ is an interpretation function which assigns to each symbol $s$ and world $w$, an interpretation $\mathcal{I}(s, w)$ satisfying the following,

1 . If $s$ is an $n$-ary function symbol, then $\mathcal{I}(s, w)$ is a function $\mathbb{D}^{n} \rightarrow \mathbb{D}$.

2. If $s$ is an $n$-ary predicate symbol, then $\mathcal{I}(s, w)$ is a function $\mathbb{D}^{n} \rightarrow\{\mathrm{tt}, \mathrm{ff}\}$.

3. If $s$ is a constant or a temporal variable, then $\mathcal{I}(s, w) \in \mathbb{D}$.

4. If $s$ is a temporal propositional letter, then $\mathcal{I}(s, w) \in\{\mathrm{tt}, \mathrm{ff}\}$.

5. If $s$ is a global symbol, then $\mathcal{I}\left(s, w_{1}\right)=\mathcal{I}\left(s, w_{2}\right)$, for all worlds $w_{1}, w_{2} \in$ $W$ i.e., its interpretation is the same in all worlds.

Semantics Given a Kripke model $\mathcal{K}$, each term $t$ is assigned a meaning on $I D$ in each world of $W$. Given an interpretation $\mathcal{I}$, a valuation $\nu$ and a world $w$; the semantics of a term is defined by induction 2 2 on its length in a standard way and is written as $\mathcal{I}_{\nu}(t, w)$. For a rigid term, the interpretation of the term is the same in all worlds.

Now we describe the semantics of the formulas. We shall write $\mathcal{K}, \nu, w \models A$ to denote that a formula $\mathrm{A}$ is satisfied in the world $w$ under the Kripke model $\mathcal{K}$ and valuation $\nu$. It can be defined by induction on formulas in a standard way with $R_{l}$ and $R_{r}$ playing the role similar to binary accessibility relation in ordinary modal logic [8]. We illustrate the cases for modal operators.

1. $\mathcal{K}, \nu, w \models \diamond_{l} A$ iff there exists $w^{\prime} \in W$ such that $R_{l}\left(w, w^{\prime}\right)$ and $\mathcal{K}, \nu, w^{\prime} \models A$

2. $\mathcal{K}, \nu, w=\diamond_{r} A$ iff there exists $w^{\prime} \in W$ such that $R_{r}\left(w, w^{\prime}\right)$ and $\mathcal{K}, \nu, w^{\prime} \models A$

We say that $\mathcal{K}$ satisfies a formula $A$ (or $A$ has a Kripke model $\mathcal{K}$ ), if there are a world $w$ and a valuation $\nu$ such that $\mathcal{K}, \nu, w \models A$. An NL formula $A$ is valid in a Kripke model $\mathcal{K}$ if for any valuation $\nu$ and world $w, \mathcal{K}, \nu, w \models A$. An NL formula $A$ is valid if $A$ is valid in every Kripke model.

A set $\Gamma$ of sentences is consistent 8 if there does not exist any finite subset $\left\{A_{1}, \ldots, A_{n}\right\}$ of $\Gamma$ such that $\vdash \neg\left(A_{1} \wedge \ldots \wedge A_{n}\right)$. If, in addition, there does 
not exist any consistent set $\Gamma^{\prime}$ such that $\Gamma^{\prime} \supset \Gamma$, then $\Gamma$ is called a maximal consistent set $(\mathrm{mcs})$.

Let $\mathbb{B}$ be a countably infinite set of symbols not occurring in the language $\mathcal{L}$. Let $\mathcal{L}^{+}$be the language obtained by adding to $\mathcal{L}$ all the symbols in $\mathbb{B}$ as rigid constants. Denote the extended proof system by $\mathrm{NL}^{+}$.

A set $\Gamma$ of sentences is said to have witnesses in $\mathbb{B}$ if for every sentence in $\Gamma$ of the form $\exists x . \phi(x)$, there exists a constant $b \in \mathbb{B}$ such that $\phi(b)$ is in $\Gamma$.

Let $Q$ be a sentence not provable in NL. Suppose $\Gamma=\{\neg Q\}$. It is easy to show that $\Gamma$ is consistent. Enumerating the sentences of $\mathcal{L}^{+}$and adding appropriate sentences to $\Gamma$ in stages, one can obtain a mcs $\Gamma^{*} \supseteq \Gamma$ in $\mathcal{L}^{+}$such that $\Gamma^{*}$ has a witness in $\mathbb{B}$ (cf. [4]). Let $\Sigma$ be the set of rigid formulas of $\Gamma^{*}$.

We shall now construct the desired Kripke model $\mathcal{K}_{\Gamma}=\left\langle W, R_{l}, R_{r}, \mathbb{D}, \mathcal{I}\right\rangle$. Let

$$
W=\{\Delta: \Delta \text { is a mcs with witnesses in } \mathbb{B} \text { and } \Delta \supseteq \Sigma\} .
$$

$W$ is non-empty since $\Gamma^{*} \in W$. The accessibility relations $R_{l}, R_{r}$ are defined as follows.

$$
R_{l}\left(\Delta_{1}, \Delta_{2}\right) \stackrel{\text { def }}{\Leftrightarrow} \diamond_{l} \Delta_{2} \subseteq \Delta_{1} \text { and } R_{r}\left(\Delta_{1}, \Delta_{2}\right) \stackrel{\text { def }}{\Leftrightarrow} \diamond_{r} \Delta_{2} \subseteq \Delta_{1} .
$$

The domain $\mathbb{D}$ is defined as follows. In $\mathbb{B}$ define a relation $\equiv$ by

$$
a \equiv b \text { iff } a=b \in \Sigma \text {. }
$$

The axioms $\mathbf{D} 1$ for equality show that $\equiv$ is an equivalence relation on $\mathbb{B}$. Let

$$
\mathbb{D}=\{[b]: b \in \mathbb{B}\}
$$

be the set of equivalence classes, where $[a]$ denotes the equivalence class containing $a$.

The interpretation function $\mathcal{I}$ is defined as follows.

1. If $v$ is a temporal variable, then $\mathcal{I}(v, \Delta)=[a]$ iff $v=a \in \Delta$

2. If $a$ is a constant, then $\mathcal{I}(a, \Delta)=[c]$ iff $a=c \in \Delta$

3 . If $f$ is an $n$-ary function symbol, then

4. If $G$ is an $n$-ary predicate symbol, then

$$
\mathcal{I}(f, \Delta)\left(\left[b_{1}\right], \ldots,\left[b_{n}\right]\right)=[c] \text { iff } f\left(b_{1}, \ldots, b_{n}\right)=c \in \Delta .
$$

$$
\mathcal{I}(G, \Delta)\left(\left[b_{1}\right], \ldots,\left[b_{n}\right]\right)=\# \text { iff } G\left(b_{1}, \ldots, b_{n}\right) \in \Delta
$$

5. If $X$ is a propositional letter, then $\mathcal{I}(X, \Delta)=\#$ iff $X \in \Delta$.

Lemma 1 ((Truth Lemma)). For any formula $A\left(x_{1}, \ldots, x_{n}\right)$, where the free variables in $A$ are among $x_{1}, \ldots, x_{n}$, for any world $\Delta \in W$ and valuation $\nu$, $\mathcal{K}_{\Gamma}, \nu, \Delta \models A\left(x_{1}, \ldots, x_{n}\right)$ iff $A\left(b_{1}, \ldots, b_{n}\right) \in \Delta$, where $\nu\left(x_{i}\right)=\left[b_{i}\right] ; 1 \leq i \leq n$.

Since $\neg Q \in \Gamma^{*}$; by Lemma 1, $\mathcal{K}_{\Gamma}, \nu, \Gamma^{*} \models \neg Q$ for any valuation $\nu$. Moreover, if a sentence $A$ is a theorem of NL then it is in $\Gamma^{*}$ and so $\mathcal{K}_{\Gamma}, \nu, \Gamma^{*} \models A$. Actually it is not required to use all the axioms in the proof of Kripke completeness [2].

\section{Completeness in Interval Models}

We now translate the Kripke world to the interval models and prove a completeness result in the interval models. 
Consider the Kripke Model $\mathcal{K}_{\Gamma}=\left\langle W, R_{l}, R_{r}, \mathbb{D}, \mathcal{I}\right\rangle$ such that $\mathcal{K}_{\Gamma}, \nu, \Gamma^{*} \models$ $\neg Q$, as build earlier. From this Kripke model through a sequence of steps, we shall construct an interval model $\mathcal{M}=\left\langle\mathbb{D}^{*}, \mathcal{I}^{*}\right\rangle$ and an interval $[a, b]$ such that $\mathcal{M}, \nu,[a, b] \models \neg Q$, for any valuation $\nu$.

Define $\mathbb{D}^{*}=\mathbb{D}$. It is quite straightforward to check that $\mathbb{D}^{*}$ satisfies all the axioms D 1 - D 4. Let $\Delta_{0} \in W$ such that $\ell=0 \in \Delta_{0}$ and $\diamond_{l} \Delta_{0} \subseteq \Gamma^{*}$. Such a $\Delta_{0}$ exists (See [2]). Recall that $\mathbb{D}$ is a set of equivalence classes (of rigid constants added to $\mathcal{L}$ ). From now on we shall not distinguish between $a$ and the equivalence class $[a]$ containing $a$. Given an interval $[a, b], a, b \in \mathbb{D}$, we shall construct a world $\Delta_{[a, b]}$ as described below.

Construction of $\Delta_{[a, b]}$ We think of the world $\Delta_{0}$ as representing 0 . We consider the following cases.

Case $1 \quad a \geq 0$.

Let $\Delta_{1}$ be a world in $W$ such that $\ell=a \in \Delta_{1}$ and $\diamond_{r}\left(\Delta_{1}\right) \subseteq \Delta_{0}$. Then $\Delta_{[a, b]}$ is a world such that $(\ell=b-a) \in \Delta_{[a, b]}$ and $\diamond_{r}\left(\Delta_{[a, b]}\right) \subseteq \Delta_{1}$.

The existence and uniqueness of such worlds can be established [2]. (Think of $\Delta_{1}$ as representing the interval $[0, a]$ which is to the right of 0 represented by $\Delta_{0}$. Then $\Delta_{[a, b]}$ represents the interval of length $(b-a)$ to the right of $\Delta_{1}$; see Figure [1])

Case $2 \quad a<0$.

Let $\Delta_{2}, \Delta_{3} \in W$ such that $\ell=-a \in \Delta_{2}$ and $\diamond_{l}\left(\Delta_{2}\right) \subseteq \Delta_{0}$. Also, $\ell=0 \in$ $\Delta_{3}$ and $\diamond_{l}\left(\Delta_{3}\right) \subseteq \Delta_{2}$.

Then, $\Delta_{[a, b]}$ is a world such that $(\ell=b-a) \in \Delta_{[a, b]}$ and $\diamond_{r}\left(\Delta_{[a, b]}\right) \subseteq \Delta_{3}$. Such a world $\Delta_{[a, b]}$ can be uniquely found [2. (Think of $\Delta_{2}$ as representing the interval $[-a, 0]$ which is to the left of 0 represented by $\Delta_{0}$. Also $\Delta_{3}$ represents the point interval $[-a,-a]$. Then $\Delta_{[a, b]}$ represents the interval of length $(b-a)$ to the right of $\Delta_{3}$; see Figure 1 ).

Now, define the function $\mathcal{I}^{*}$ as, $\mathcal{I}^{*}(s,[a, b])=\mathcal{I}\left(s, \Delta_{[a, b]}\right)$, for any symbol $s$. From the definition of $\mathcal{I}$ it follows that $\mathcal{I}^{*}(\ell,[a, b])=\mathcal{I}\left(\ell, \Delta_{[a, b]}\right)=b-a$.

We now need the following lemma which can be proved by taking induction on formulas ( $c f .[2])$.

Lemma 2. For any interval $[a, b]$, valuation $\nu$ and formula $A$ $\mathcal{M}, \nu,[a, b] \models A \quad$ iff $\mathcal{K}, \nu, \Delta_{[a, b]} \models A$.

We have $\mathcal{K}, \nu, \Gamma^{*} \models \neg Q$. Now it can be shown that (see [2]) for some $c \geq$ $0, \quad \Gamma^{*}=\Delta_{[0, c]}$, for any valuation $\nu$. Thus we have $\mathcal{K}, \nu, \Delta_{[0, c]} \models \neg Q$, for all valuation $\nu$. Hence by Lemma 2, $\mathcal{M}, \nu,[0, c] \models \neg Q$, for any valuation $\nu$. Thus $Q$ is not valid. Hence we have,

Theorem 2 ((Completeness of NL)). If a sentence $Q$ is valid in interval models, then $Q$ is provable in $N L$.

\section{Discussion}

A complete axiomatic system for a first-order interval logic with two neighbourhood modalities has been presented in this paper. Barua and Zhou [2] have 


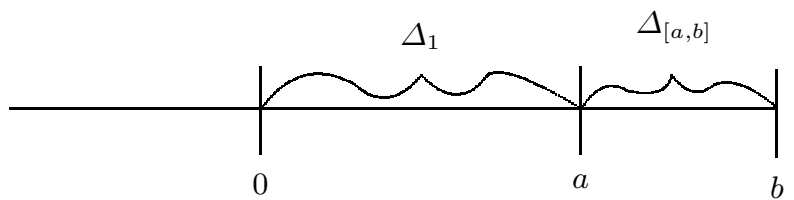

Case 1: $a \geq 0$

$\Delta_{0}$

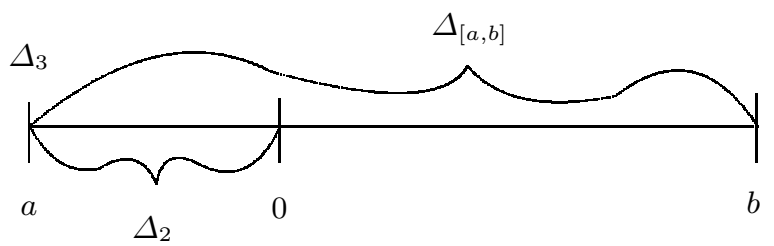

Case 2: $a<0$

$\Delta_{0}$

Fig. 1. Construction of $\Delta_{[a, b]}$

extended NL by introducing two more modalities in the upward and downward directions and have proposed a two-dimensional neighbourhood logic $N L^{2}$. They have proved a completeness result in $N L^{2}$ using the same construction. Their work suggests that it may be possible to obtain a proof system of Neighbourhood Logic in any dimension using the same technique. The logic of $N L^{2}$ can be used to specify the behaviour of the real-time systems where timeless computation is taken into account [3].

In 14] NL has been extended to obtain a Duration Calculus (DC) where temporal variables are expressed in the form of integrals (durations) of state variables. It is interesting to note that the proof system of DC is relatively complete, i.e. it is complete provided all valid NL formulas (with time domain and valuation domain taken to be reals) are considered as axioms in the proof system of DC (cf. [14]).

Applications of NL (and $\mathrm{NL}^{2}$ ) are being investigated. In [13] NL is combined with a linear temporal logic to give a real-time semantics for an OCCAM-like language, where timeless computation was assumed. Further NL is applied for Interval Algebra in the area of Artificial Intelligence [12].

\section{References}

1. Alur R., Courcoubetis C., Henzinger T., Ho P-H.: Hybrid Automata: An algorithmic approach to the specification and verification of Hybrid systems, in Hybrid Systems, R. L. Grossman, A. Nerode, A. P. Ravn and H. Rischel (Eds.), LNCS 736, pp. 209-229, Springer-Verlag, 1993. 
2. Barua Rana, Zhou Chaochen: Neighbourhood Logics: NL and $\mathrm{NL}^{2}, U N U / I I S T$ Report no. 120, 1997. I

3. Berry Gérard, Gonthier Georges: The Esterel Synchronous Programming Language: Design, Semantics and Implementation, in Science of Computer Programming, vol. 19, pp. 87-152, Elsevier, 1992.

4. Dutertre B.: Complete Proof Systems for First Order Interval Logic, Tenth Annual IEEE Symp. on Logic in Computer Science, pp. 36-43, IEEE Press, 1995.

5. Halpern J., Shoham Y.: A Propositional Modal Logic of Time Intervals, Journal of the ACM 38 (4) pp. 935-962, 1991. Also appeared in Proceedings of the First IEEE Symposium on Logic in Computer Science, pp. 279-292, Computer Society Press, 1986.

6. Hansen Michael, Zhou Chaochen: Duration Calculus: Logical Foundations, To appear in Formal Aspects of Computing.

7. Humberstone: Interval semantics for Tense Logic, Jour of Phil. Logic, 8, 1979.

8. Hughes G. E., Creswell M. J.: An introduction to Modal Logic, Routledge, 1990.

9. Lamport L.: Hybrid systems in TLA ${ }^{+}$, in in Hybrid Systems, R. L. Grossman, A. Nerode, A. P. Ravn and H. Rischel (Eds.), LNCS 736, pp. 77-102, Springer-Verlag, 1993.

10. Manna Z., Pnueli A.: Verifying hybrid systems, in Hybrid Systems, R. L. Grossman, A. Nerode, A. P. Ravn and H. Rischel (Eds.), LNCS 736, pp. 4-35, Springer-Verlag. 1993.

11. Moszkowski B.: A Temporal Logic for Multilevel Reasoning about Hardware, IEEE Computer 18 (2) pp. 10-19, 1985.

12. Pujari Arun K: Neighbourhood Logic \& Interval Algebra. UNU/IIST Report no. 116, 1997.

13. Qiu Zongyan, Zhou Chaochen: A Combination of Interval Logic and Linear Temporal Logic. UNU/IIST Report no. 123, 1997 (accepted by PROCOMET'98).

14. Roy Suman, Zhou Chaochen: Notes in Neighbourhood Logic, UNU/IIST Report no. 97, 1997.

15. Shoenfield J., Mathematical Logic, Addison-Wesley, Reading, Mass., 1967.

16. Venema Y.: Expressiveness and Completeness of an Interval Tense Logic, Notre Dame Journal of Formal Logic, Vol. 31, No. 4, pp. 529-547, 1990.

17. Venema Y.: A Modal Logic for Chopping Intervals, Journal of Logic and Computation, Vol. 1, pp. 453-476, Oxford University Press, 1991.

18. Zhou Chaochen, Hansen Michael R.: An Adequate First Order Interval Logic. UNU/IIST Report No. 91, Revised report, December 1996.

19. Zhou Chaochen, Hoare C. A. R., Ravn A. P.: A Calculus of Durations, Information Processing Letters, Vol. 40, No. 5, pp. 269-276, 1991.

20. Zhou Chaochen, Ravn A. P., Hansen Michael R.: An extended duration calculus for hybrid systems, in Hybrid Systems, R. L. Grossman, A. Nerode, A. P. Ravn and H. Rischel (Eds.), LNCS 736, pp. 36-59, Springer-Verlag, 1993. 